\title{
UWB Transmitted Reference Signaling Schemes - Part I: Performance Analysis
}

\author{
Tony Q.S. Quek and Moe Z. Win \\ Laboratory for Information \& Decision Systems (LIDS) \\ Massachusetts Institute of Technology \\ Cambridge, MA 02139, USA \\ Email : \{qsquek,moewin\}@mit.edu
}

\author{
Davide Dardari \\ IEIIT-CNR, DEIS, University of Bologna \\ Bologna, Italy \\ Email: ddardari@deis.unibo.it
}

\begin{abstract}
Transmitted-Reference (TR) signaling, in conjunction with an autocorrelation receiver (AcR), offers a lowcomplexity alternative to Rake reception. Due to its simplicity, there is renewed interest in TR signaling for ultrawide bandwidth (UWB) systems. Different variations of TR signaling have been proposed and investigated, including differential TR (DTR) signaling and noise averaging at the AcR. This paper provides performance analysis of various TR schemes by developing an analytical framework based on the sampling expansion approach. Specifically, we derive uncoded bit error probability (BEP) of different TR signaling schemes such as DTR and noise averaging at the AcR for a broad class of fading channels.
\end{abstract}

\section{INTRODUCTION}

Recently, there has been renewed interest in utilizing ultrawide bandwidth (UWB) spread-spectrum communications for future military, homeland security and commercial applications. UWB systems involve the transmission of a train of extremely narrow pulses by employing either time-hopping (TH) or direct sequence (DS) techniques for multiple access and pulse position modulation (PPM) or pulse amplitude modulation (PAM) for data transmission [1]. The key motivation for using UWB systems is the ability to highly resolve multipath components, as well as the availability of technology to implement and generate UWB signals with relatively low complexity. These fine delay resolution properties make UWB radio a viable candidate for communications in dense multipath environments such as short-range or indoor wireless communications [2]-[4].

This paper considers a signaling scheme referred to as transmitted-reference (TR) signaling [5]-[7]. TR signaling involves the transmission of a reference and data signal pair, separated either in time [6], [7] or in frequency [8]. In order for this pair of separated signals to experience the same channel, either the time separation must be less than the channel coherence time, or the frequency separation must be less than the channel coherence bandwidth. The receiver can simply be an autocorrelation receiver (AcR), which may also be modified to include noise averaging for better performance [9]-[12]. Since TR signaling allocates a significant part of the symbol energy by transmitting reference pulses, differential encoding over consecutive symbols can also be used to alleviate this communication resource wastage problem. This alternative TR signaling is referred to as differential transmitted-reference
(DTR) signaling [10], [13]. The performance analysis of TR and DTR signaling published in literature is based on averaging numerically the conditional bit error probability (BEP) via a quasi-analytical/simulation approach [10] or quasi analytical/experimental approach [9], whose results rely on either simulation parameters or experimental data. Therefore, there is a need to provide an analytical tool to compare TR and DTR signaling schemes. We aim to provide an analytical framework to derive BEP of these schemes in dense resolvable multipath channels. We adopt the sampling expansion approach, which is an extension of our previous work [11], [12]. We derive the BEP for TR and DTR signaling when AcR or modified AcR is used for a broad class of fading channels in dense resolvable multipath channels.

The remainder of the paper is organized as follows. Section II presents the system and channel models for both TR and DTR signaling schemes of a single link system. In Section III, a unified BEP analysis of TR and DTR signaling based on the sampling expansion approach is developed. To illustrate our proposed methodology, we consider Nakagami- $m$ fading channels and present numerical results in Section IV. Finally, Section V comprises concluding remarks.

\section{Signal AND Channel Models}

\section{A. Transmitted-Reference}

In TR signaling, the transmitted signal for a single user can be decomposed into a reference signal block $b_{\mathrm{r}}(t)$ and a data modulated signal block $b_{\mathrm{d}}(t)$ as given by

$$
s_{\mathrm{TR}}(t)=\sum_{i} b_{\mathrm{r}}\left(t-i N_{\mathrm{s}} T_{\mathrm{f}}\right)+d_{i} b_{\mathrm{d}}\left(t-i N_{\mathrm{s}} T_{\mathrm{f}}\right),
$$

where $T_{\mathrm{f}}$ is the average repetition period, $d_{i} \in\{-1,1\}$ is the data symbol, and each block has symbol duration $N_{\mathrm{s}} T_{\mathrm{f}}$ [9]-[12] as shown in Fig. 1. Each block, containing $N_{\mathrm{s}} / 2$ transmitted signal pulses, can be written as ${ }^{1}$

$$
b_{\mathrm{r}}(t)=\sum_{j=0}^{\frac{N_{\mathrm{s}}}{2}-1} \sqrt{E_{\mathrm{p}}} a_{j} p\left(t-j 2 T_{\mathrm{f}}-c_{j} T_{\mathrm{p}}\right),
$$

\footnotetext{
${ }^{1}$ Note that other combination of data and reference pulses is also possible. Here, without loss of generality, we have adopted the conventional TR signaling where the number of reference and data pulses are equal for simplicity [9].
} 


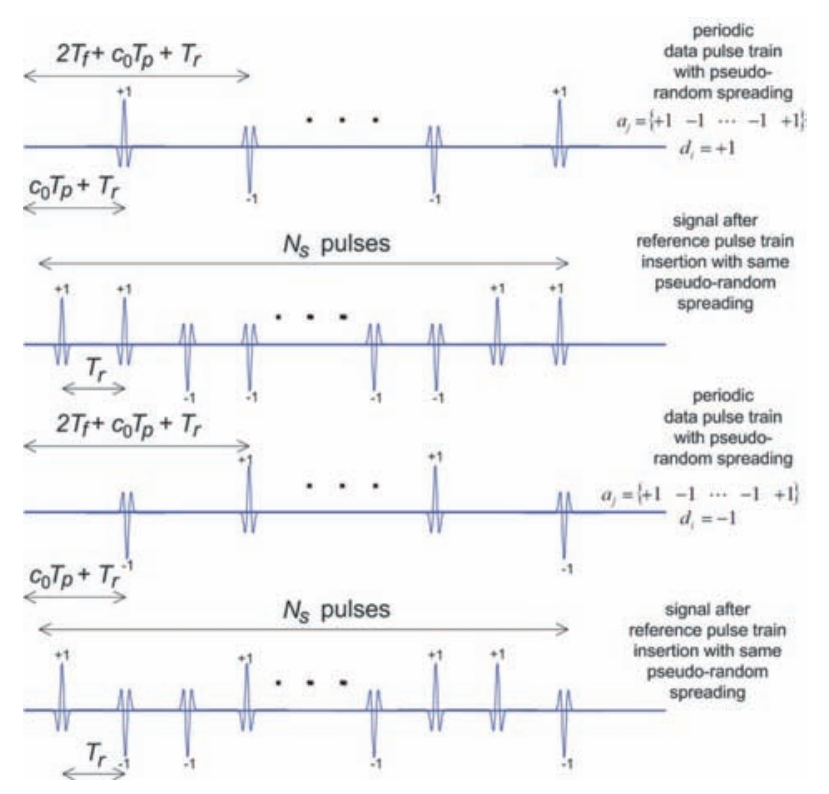

Fig. 1. TR signaling.

$$
b_{\mathrm{d}}(t)=\sum_{j=0}^{\frac{N_{\mathrm{s}}}{2}-1} \sqrt{E_{\mathrm{p}}} a_{j} p\left(t-j 2 T_{\mathrm{f}}-c_{j} T_{\mathrm{p}}-T_{\mathrm{r}}\right),
$$

where $p(t)$ is the normalized signal pulse with duration $T_{\mathrm{p}}$ and $\int_{-\infty}^{+\infty}|p(t)|^{2} d t=1$. The energy of the transmitted pulse is then $E_{\mathrm{p}}=E_{\mathrm{s}} / N_{\mathrm{s}}$, and symbol energy is $E_{\mathrm{s}}$. In our case of binary signaling, $E_{\mathrm{s}}=E_{\mathrm{b}}$, where $E_{\mathrm{b}}$ is the energy per bit. To enhance the robustness of TR systems to interference as well as to allow multiple access, DS and/or TH spread spectrum techniques can be used as shown in (2). In DS signaling, $\left\{a_{j}\right\}$ is the bipolar pseudo-random sequence. In TH signaling, $\left\{c_{j}\right\}$ is the pseudo-random TH sequence, where $c_{j}$ is an integer in the range $0 \leq c_{j}<N_{\mathrm{h}}$, and $N_{\mathrm{h}}$ is the maximum allowable integer shift. The duration of the received UWB pulse is $T_{\mathrm{g}}=T_{\mathrm{p}}+T_{\mathrm{d}}$, where $T_{\mathrm{d}}$ is the maximum excess delay of the channel. To preclude inter-symbol interference (ISI) and intra-symbol interference (i.s.i.) ${ }^{2}$, we assume that $T_{\mathrm{r}} \geq T_{\mathrm{g}}$ and $N_{\mathrm{h}} T_{\mathrm{p}}+T_{\mathrm{r}} \leq 2 T_{\mathrm{f}}-T_{\mathrm{g}}$, where $T_{\mathrm{r}}$ is the time separation between each pair of data and reference pulses such that these received pulses will not overlap. Note that the interpulse delay between each data-modulated monocycle and its corresponding reference monocycle is given by $T_{\mathrm{r}}$.

\section{B. Differential Transmitted-Reference}

In DTR signaling, the transmitted signal for a single user is given by

$$
s_{\mathrm{DTR}}(t)=\sum_{i} e_{i} b\left(t-i N_{\mathrm{s}} T_{\mathrm{f}}\right)
$$

where $b(t)$ is the block-modulated signal with symbol duration $N_{\mathrm{s}} T_{\mathrm{f}}$. The data symbol $d_{i}$ is now differentially encoded such that $e_{i}=e_{i-1} d_{i}$, where $d_{i}= \pm 1$. Within each $b(t)$-shaped

\footnotetext{
${ }^{2}$ ISI and i.s.i. may not always be negligible due to constraints on $T_{\mathrm{f}}$ and data-rate requirements. In this case, our results will serve as a lower bound.
}

block, there are $N_{\mathrm{s}}$ transmitted signal pulses and it can be written as

$$
b(t)=\sum_{j=0}^{N_{\mathrm{s}}-1} \sqrt{E_{\mathrm{p}}} a_{j} p\left(t-j T_{\mathrm{f}}-c_{j} T_{\mathrm{p}}\right),
$$

where $\left\{a_{j}\right\}$ and $\left\{c_{j}\right\}$ are the DS and TH sequences that provide the multiple access capability of DTR systems. The length of $\left\{a_{j}\right\}$ is now $N_{\mathrm{s}}$. The TH sequence is pseudorandom with the range $0 \leq c_{j}<N_{\mathrm{h}}$, where $N_{\mathrm{h}}$ satisfies $T_{\mathrm{f}} \geq\left(N_{\mathrm{h}}-1\right) T_{\mathrm{p}}+T_{\mathrm{g}}$ to preclude ISI and i.s.i. The channel is assumed to be constant over two symbols in order to use differential encoding over every two symbols. ${ }^{3}$

\section{Channel model}

The received signal for TR signaling is $r(t)=\left(h * s_{\mathrm{TR}}\right)(t)+$ $n(t)$, where $h(t)$ is the impulse response of the channel and $n(t)$ is zero-mean, white Gaussian noise with two-sided power spectral density $N_{0} / 2$. Note that similar equation also applies to DTR signaling by replacing $s_{\mathrm{TR}}(t)$ with $s_{\mathrm{DTR}}(t)$. The channel impulse response being modelled as linearly timeinvariant can be written as $h(t)=\sum_{l=1}^{L} \alpha_{l} \delta\left(t-\tau_{l}\right)$ where $\alpha_{l}$ and $\tau_{l}$ denote respectively the attenuation and delay of $l$-TH path, and $L$ is the number of resolvable multipath components. We can also express $\alpha_{l}=\left|\alpha_{l}\right| \exp \left(j \phi_{l}\right)$, where $\phi_{l}=0$ or $\pi$ with equal probability. As in [2], [3], we consider the resolvable channel, i.e., $\left|\tau_{l}-\tau_{j}\right| \geq T_{\mathrm{p}}, \forall l \neq j$, where $\tau_{l}=\tau_{1}+(l-1) T_{\mathrm{p}}$. However, depending on whether the UWB channel is uncorrelated scattering [3] or correlated scattering [4], $\left\{\alpha_{l}\right\}$ can be statistically independent or correlated random variables (r.v's).
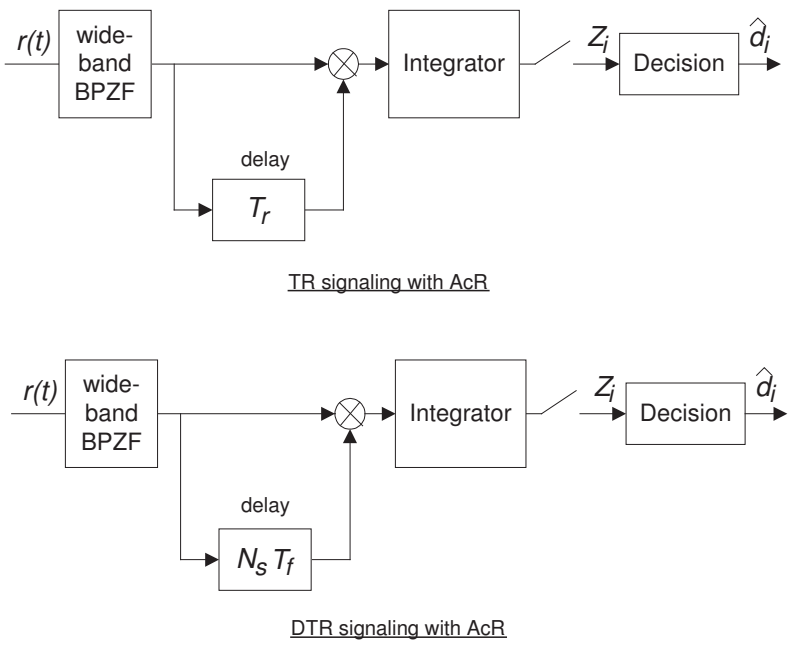

Fig. 2. AcR for TR and DTR signaling.

\section{RECEIVER MODEL}

\section{A. Autocorrelation Receiver}

As shown in Fig. 2, the AcR first passes the received signal through an ideal bandpass zonal filter (BPZF), with

${ }^{3}$ We can relax this assumption by encoding the data differentially across pairs of pulses. 
bandwidth $W$ and center frequency $f_{\mathrm{c}}$ around the signal band to eliminate the out-of-band noise. If $W$ is wide enough, then the signal spectrum will pass undistorted through the BPZF. Consequently, the ISI and i.s.i. caused by filtering will be negligible. The filtered received signal is then passed through a correlator with integration interval $T\left(T_{\mathrm{p}} \leq T \leq T_{\mathrm{g}}\right)$, as shown in Fig. 2, to collect the received signal energy. The integration interval $\mathrm{T}$ determines the number of multipath components (or equivalently, the amount of energy) captured by the receiver as well as the amount of noise and interference accumulation. Without loss of generality, we consider the detection of the data symbol at $i=0$. In addition, we assume perfect synchronization at the receiver. The decision statistics generated at the AcR for TR and DTR signaling are given respectively by

$$
Z_{\mathrm{TR}}=\sum_{j=0}^{\frac{N_{\mathrm{s}}}{2}-1} \int_{j 2 T_{\mathrm{f}}+T_{\mathrm{r}}+c_{j} T_{\mathrm{p}}}^{j 2 T_{\mathrm{f}}+T_{\mathrm{r}}+c_{j} T_{\mathrm{p}}+T} \widetilde{r}_{\mathrm{TR}}(t) \widetilde{r}_{\mathrm{TR}}\left(t-T_{\mathrm{r}}\right) d t,
$$

and

$Z_{\mathrm{DTR}}=\sum_{j=0}^{N_{\mathrm{s}}-1} \int_{j T_{\mathrm{f}}+c_{j} T_{\mathrm{p}}}^{j T_{\mathrm{f}}+c_{j} T_{\mathrm{p}}+T} \widetilde{r}_{\mathrm{DTR}}(t) \widetilde{r}_{\mathrm{DTR}}\left(t-N_{\mathrm{s}} T_{\mathrm{f}}\right) d t$,

where $\widetilde{r}_{\mathrm{TR}}(t)=\left(h * s_{\mathrm{TR}}\right)(t)+\widetilde{n}(t)$ and $\widetilde{r}_{\mathrm{DTR}}(t)=(h *$ $\left.s_{\mathrm{DTR}}\right)(t)+\widetilde{n}(t)$. Note that $\widetilde{n}(t)$ is a zero-mean, Gaussian random process with autocorrelation function

$$
R_{\widetilde{n}}(\tau)=W N_{0} \operatorname{sinc}(W \tau) \cos \left(2 \pi f_{\mathrm{c}} \tau\right) .
$$

When $W \gg 1 / T_{\mathrm{g}}, R_{\widetilde{n}}(t-u)$ in (7) is approximately equal to zero for $|t-u| \geq T_{\mathrm{g}}$. Hence, the noise samples separated by more than $T_{\mathrm{g}}$ or a multiple of $1 / W$ can assume to be statistically independent.

\section{B. Modified Autocorrelation Receiver}

The AcR performance can be improved for TR and DTR signaling by averaging respectively over $N_{\mathrm{s}} / 2$ and $N_{\mathrm{s}}$ received reference pulses from the previous symbol respectively, to be used as an estimate of the channel [9]-[12]. This approach requires that the channel remains constant over two symbols. The decision statistics of this modified AcR for TR and DTR signaling are given respectively by

$$
\begin{aligned}
Z_{\mathrm{ATR}}= & \sum_{j=0}^{\frac{N_{\mathrm{s}}}{2}-1} a_{j} \int_{j 2 T_{\mathrm{f}}+T_{\mathrm{r}}+c_{j} T_{\mathrm{p}}}^{j 2 T_{\mathrm{f}}+T_{\mathrm{r}}+c_{j} T_{\mathrm{p}}+T} \tilde{r}_{\mathrm{TR}}(t) \\
& \left(\frac { 2 } { N _ { \mathrm { s } } } \sum _ { k = - j } ^ { \frac { N _ { \mathrm { s } } } { 2 } - 1 - j } a _ { j + k } \widetilde { r } _ { \mathrm { TR } } \left(t-\left(N_{\mathrm{s}}-2 k\right) T_{\mathrm{f}}\right.\right. \\
& \left.\left.-\left(c_{j}-c_{j+k}\right) T_{\mathrm{p}}-T_{\mathrm{r}}\right)\right) d t
\end{aligned}
$$

and

$$
\begin{aligned}
Z_{\mathrm{ADTR}}= & \sum_{j=0}^{N_{\mathrm{s}}-1} a_{j} \int_{j T_{\mathrm{f}}+c_{j} T_{\mathrm{p}}}^{j T_{\mathrm{f}}+c_{j} T_{\mathrm{p}}+T} \widetilde{r}_{\mathrm{DTR}}(t) \\
& \left(\frac { 1 } { N _ { \mathrm { s } } } \sum _ { k = - j } ^ { N _ { \mathrm { s } } - 1 - j } a _ { j + k } \widetilde { r } _ { \mathrm { DTR } } \left(t-\left(N_{\mathrm{s}}-k\right) T_{\mathrm{f}}\right.\right. \\
& \left.\left.-\left(c_{j}-c_{j+k}\right) T_{\mathrm{p}}\right)\right) d t .
\end{aligned}
$$

Next, we develop an analytical framework based on the sampling expansion approach to provide a unified performance analysis of TR and DTR systems in dense multipath channels [11], [12]. Our methodology does not adopt the Gaussian approximation that is commonly used to derive the conditional BEP in terms of the Gaussian $Q$-function. The methodology still allows us to obtain BEP of TR and DTR systems for a broad class of fading channels.

\section{Performance Analysis}

\section{A. Transmitted-Reference}

It can be shown that $Z_{\mathrm{TR}}$ in (5) can be written as [11], [12]

$$
\begin{aligned}
Z_{\mathrm{TR}} & \\
=\sum_{j=0}^{\frac{N_{\mathrm{s}}}{2}-1} & \int_{0}^{T}\left(\breve{b}_{\mathrm{r}}\left(t+j 2 T_{\mathrm{f}}+c_{j} T_{\mathrm{p}}\right)+\widetilde{n}\left(t+j 2 T_{\mathrm{f}}+c_{j} T_{\mathrm{p}}\right)\right) \\
& \times\left(d_{0} \breve{b}_{\mathrm{d}}\left(t+j 2 T_{\mathrm{f}}+c_{j} T_{\mathrm{p}}+T_{\mathrm{r}}\right)\right. \\
& \left.+\widetilde{n}\left(t+j 2 T_{\mathrm{f}}+c_{j} T_{\mathrm{p}}+T_{\mathrm{r}}\right)\right) d t
\end{aligned}
$$

where $\breve{b}_{\mathrm{r}}(t) \triangleq\left(b_{\mathrm{r}} * h * h_{\mathrm{ZF}}\right)(t), \breve{b}_{\mathrm{d}}(t) \triangleq\left(b_{\mathrm{d}} * h * h_{\mathrm{ZF}}\right)(t)$, and $h_{\mathrm{ZF}}(t)$ is the impulse response of the BPZF. Note that if the symbol duration is less than the coherence time, all pairs of separated pulses will experience the same channel, implying that $\breve{b}_{\mathrm{r}}\left(t+j 2 T_{\mathrm{f}}+c_{j} T_{\mathrm{p}}\right)=\breve{b}_{\mathrm{d}}\left(t+j 2 T_{\mathrm{f}}+c_{j} T_{\mathrm{p}}+T_{\mathrm{r}}\right)$ for all $t \in(0, T)$ and $c_{j}$. In this case, we can significantly simplify the expression in (10) as follows:

$$
\begin{aligned}
Z_{\mathrm{TR}} & =\sum_{j=0}^{\frac{N_{\mathrm{s}}}{2}-1} \int_{0}^{T}\left(w_{j}(t)+\eta_{1, j}(t)\right)\left(d_{0} w_{j}(t)+\eta_{2, j}(t)\right) d t \\
& =\sum_{j=0}^{\frac{N_{\mathrm{s}}}{2}-1} U_{j}
\end{aligned}
$$

where we have used $w_{j}(t) \triangleq \breve{b}_{\mathrm{r}}\left(t+j 2 T_{\mathrm{f}}+c_{j} T_{\mathrm{p}}\right)=$ $\sqrt{E_{\mathrm{p}}} a_{j} \sum_{l=1}^{L} \alpha_{l} p\left(t-\tau_{l}\right), \eta_{1, j}(t) \triangleq \widetilde{n}\left(t+j 2 T_{\mathrm{f}}+c_{j} T_{\mathrm{p}}\right)$ and $\eta_{2, j}(t) \triangleq \widetilde{n}\left(t+j 2 T_{\mathrm{f}}+c_{j} T_{\mathrm{p}}+T_{\mathrm{r}}\right)$ defined over the interval $[0, T]$.

Since the received signal is a real bandpass signal of bandwidth $W$, one can think of it in terms of its complex baseband equivalent model. In this case, the signal is complex and bandlimited to $W / 2$. The Sampling Theorem then states that this must be sampled at a sampling frequency greater than or equal to $W$. This gives $W T$ complex dimensions or $2 W T$ 
real dimensions [14]. Following this sampling approach [11], [12], we can then represent $U_{j}$ as

$$
\begin{gathered}
U_{j}=\frac{1}{W} \sum_{m=1}^{2 W T}\left(d_{0} w_{j, m}^{2}+w_{j, m} \eta_{2, j, m}+d_{0} w_{j, m} \eta_{1, j, m}\right. \\
\left.+\eta_{1, j, m} \eta_{2, j, m}\right),
\end{gathered}
$$

where the $m$-th sample of $w_{j}(t), \eta_{1, j}(t)$ and $\eta_{2, j}(t)$ in (11) are respectively $w_{j, m}, \eta_{1, j, m}$, and $\eta_{2, j, m}$ in the interval $[0, T]$. Note that since the noise samples are taken at least $T_{\mathrm{g}}$ apart, they are essentially independent, regardless of $c_{j}$. Hence, no further assumption on $c_{j}$ is required in our analysis. We further observe that $U_{j}$ is simply the integrator output of the $j$-th received modulated monocycle.

Due to the statistical symmetry of $U_{j}$ with respect to $d_{0}$, we simply need to calculate the BEP conditioned on $d_{0}=+1$. Hence, conditioned on $d_{0}$, we can express (12) in the form of a summation of squares

$$
\begin{aligned}
& U_{j \mid d_{0}=+1}=\sum_{m=1}^{2 W T}\left[\left(\frac{1}{\sqrt{W}} w_{j, m}+\beta_{1, j, m}\right)^{2}-\beta_{2, j, m}^{2}\right], \\
& U_{j \mid d_{0}=-1}=\sum_{m=1}^{2 W T}\left[-\left(\frac{1}{\sqrt{W}} w_{j, m}-\beta_{2, j, m}\right)^{2}+\beta_{1, j, m}^{2}\right],
\end{aligned}
$$

where $\beta_{1, j, m}=\frac{1}{2 \sqrt{W}}\left(\eta_{2, j, m}+\eta_{1, j, m}\right), \quad \beta_{2, j, m}=$ $\frac{1}{2 \sqrt{W}}\left(\eta_{2, j, m}-\eta_{1, j, m}\right)$, and these are statistically independent Gaussian r.v.'s with variance $\sigma_{\mathrm{TR}}^{2}=\frac{N_{0}}{4}$. Recall from the definition of $w_{j}(t)$, the contribution of the sequence $\left\{a_{j}\right\}$ is embedded inside $w_{j, m}$. From (13), we can observe that when conditioned on the channel, $U_{j \mid d_{0}=+1}$ has the same probability density functions (pdfs) for $a_{j}=+1$ and $a_{j}=-1$. Due to this symmetry and $a_{j}$ being equally probable, we can assume that $a_{j}=+1$ in the following analysis without loss of generality. For notational simplicity, we define the normalized r.v.'s $Y_{1}$, $Y_{2}, Y_{3}$, and $Y_{4}$ as

$$
\begin{aligned}
& Y_{1} \triangleq \frac{1}{2 \sigma_{\mathrm{TR}}^{2}} \sum_{j=0}^{\frac{N_{\mathrm{s}}}{2}-1} \sum_{m=1}^{2 W T}\left(\frac{1}{\sqrt{W}} w_{j, m}+\beta_{1, j, m}\right)^{2}, \\
& Y_{2} \triangleq \frac{1}{2 \sigma_{\mathrm{TR}}^{2}} \sum_{j=0}^{\frac{N_{\mathrm{s}}}{2}-1} \sum_{m=1}^{2 W T} \beta_{2, j, m}^{2}, \\
& Y_{3} \triangleq \frac{1}{2 \sigma_{\mathrm{TR}}^{2}} \sum_{j=0}^{\frac{N_{\mathrm{s}}}{2}-1} \sum_{m=1}^{2 W T}\left(\frac{1}{\sqrt{W}} w_{j, m}-\beta_{2, j, m}\right)^{2}, \\
& Y_{4} \triangleq \frac{1}{2 \sigma_{\mathrm{TR}}^{2}} \sum_{j=0}^{\frac{N_{\mathrm{s}}}{2}-1} \sum_{m=1}^{2 W T} \beta_{1, j, m}^{2} .
\end{aligned}
$$

Conditioned on the channel, $Y_{1}$ and $Y_{3}$ are noncentral chisquared r.v.'s with $N_{\mathrm{s}} W T$ degrees of freedom, whereas $Y_{2}$ and $Y_{4}$ are central chi-squared r.v.'s with the same degrees of freedom as $Y_{1}$ and $Y_{3}$. Both $Y_{1}$ and $Y_{3}$ have the same non- centrality parameter given by

$$
\mu_{\mathrm{TR}}=\frac{1}{2 \sigma_{\mathrm{TR}}^{2}} \sum_{j=0}^{\frac{N_{\mathrm{s}}}{2}-1} \int_{0}^{T} w_{j}^{2}(t) d t=\frac{E_{\mathrm{s}}}{N_{0}} \sum_{l=1}^{L_{\mathrm{CAP}}} \alpha_{l}^{2},
$$

where $L_{\mathrm{CAP}} \triangleq\left\lceil\min \left\{W T, W T_{\mathrm{g}}\right\}\right\rceil$ denotes the actual number of multipath components captured by the AcR. Note that $\gamma_{\mathrm{TR}}=\mu_{\mathrm{TR}} / 2$ is the instantaneous received SNR of TR signaling with AcR [11], [12]. The pdfs of $Y_{1}$ and $Y_{2}$ conditioned on $\gamma_{\mathrm{TR}}$ are given by

$$
\begin{aligned}
& f_{Y_{1} \mid \gamma_{\mathrm{TR}}}\left(y_{1}\right)=f_{\mathrm{NC}}\left(y_{1}, \mu_{\mathrm{TR}}, q_{\mathrm{TR}}\right), \\
& f_{Y_{2} \mid \gamma_{\mathrm{TR}}}\left(y_{2}\right)=f_{\mathrm{C}}\left(y_{2}, q_{\mathrm{TR}}\right),
\end{aligned}
$$

where $q_{\mathrm{TR}}=\frac{N_{\mathrm{s}} W T}{2}$. We have defined the following pdfs for notational convenience

$$
\begin{aligned}
f_{\mathrm{NC}}(y, \mu, n) \triangleq e^{-(y+\mu)}\left(\frac{y}{\mu}\right)^{\frac{(n-1)}{2} I_{n-1}}(2 \sqrt{y \mu}), & y \geq 0 \\
f_{\mathrm{C}}(y, n) \triangleq \frac{y^{(n-1)}}{(n-1) !} \exp (-y), & y \geq 0
\end{aligned}
$$

where $I_{n-1}(\cdot)$ is the $(n-1)$-th order Bessel function of the first kind, and $f_{\mathrm{NC}}(y, \mu, n)$ and $f_{\mathrm{C}}(y, n)$ are respectively the pdfs of the noncentral and central chi-squared r.v.'s with $2 n$ degrees of freedom and non-centrality parameter $\mu$ [15]. Using (16) and (17), the BEP of TR signaling with AcR is given by

$$
\begin{aligned}
P_{\mathrm{e}, \mathrm{TR}} & =\mathbb{P}\left\{Z_{\mathrm{TR}} \leq 0 \mid d_{0}=+1\right\} \\
& =\mathbb{E}_{\gamma_{\mathrm{TR}}}\left\{\mathbb{P}\left\{Y_{1}<Y_{2} \mid d_{0}=+1\right\}\right\} \\
& =\frac{1}{2^{q_{\mathrm{TR}}}}\left[\left.\sum_{i=0}^{q_{\mathrm{TR}}-1}\left(\frac{(-j)^{i}}{i !}\right) \frac{d^{i}}{d v^{i}} \psi_{\gamma_{\mathrm{TR}}}(j v)\right|_{j v=-1}\right. \\
& \left.\sum_{k=i}^{q_{\mathrm{TR}}-1} \frac{1}{2^{k}} \frac{\left(k+q_{\mathrm{TR}}-1\right) !}{(k-i) !\left(q_{\mathrm{TR}}+i-1\right) !}\right] \\
& \triangleq P_{\mathrm{e}}\left(\psi_{\gamma_{\mathrm{TR}}}(j v), q_{\mathrm{TR}}\right),
\end{aligned}
$$

where $\psi_{\gamma_{\mathrm{TR}}}(j v) \triangleq \mathbb{E}\left\{e^{j v \gamma_{\mathrm{TR}}}\right\}$ is the characteristic function (CF) of $\gamma_{\mathrm{TR}}$ and $P_{\mathrm{e}}\left(\psi_{\gamma_{\mathrm{TR}}}(j v), q_{\mathrm{TR}}\right)$ is defined for convenience. Under the resolvable multipath and uncorrelated scattering assumption [3], multipath components are statistically independent and $\psi_{\gamma_{\mathrm{TR}}}(j v)=\prod_{l=1}^{L_{\mathrm{CAP}}} \psi_{l}\left(\frac{E_{\mathrm{s}}}{2 N_{0}} j v\right)$, where $\psi_{l}(j v)$ is the CF of $\alpha_{l}^{2}$ and it is known in closed form for a wide range of channel fading statistics [15]. When the uncorrelated scattering assumption is no longer valid, multipath components are correlated [4]. In this case, the CF of $\gamma_{\mathrm{TR}}$ can then be found by using eigenvalue decomposition and partial fraction expansion [16], [17]. The detailed derivation of (18) can be found in [11], [12].

Next, we extend the above analysis to derive the BEP of TR signaling with modified AcR [11], [12]. The non-centrality parameter of $Y_{1}$ in (14) is given by

$$
\mu_{\mathrm{ATR}}=\frac{2}{1+\frac{2}{N_{\mathrm{s}}}}\left(\frac{E_{\mathrm{s}}}{N_{0}} \sum_{l=1}^{L_{\mathrm{CAP}}} \alpha_{l}^{2}\right) .
$$


As shown in [11], [12], the BEP of TR signaling with modified AcR becomes

$$
P_{\mathrm{e}, \mathrm{ATR}}=P_{\mathrm{e}}\left(\psi_{\gamma_{\mathrm{ATR}}}(j v), q_{\mathrm{TR}}\right),
$$

where the instantaneous received SNR of TR signaling with modified AcR is given by $\gamma_{\mathrm{ATR}}=\mu_{\mathrm{ATR}} / 2$.

\section{B. Differential Transmitted-Reference}

Following an approach similar to the case of TR signaling, we can represent $U_{j}$ as

$$
\begin{gathered}
U_{j}=\frac{1}{W} \sum_{m=1}^{2 W T}\left(d_{0} w_{j, m}^{2}+e_{-1} w_{j, m} \eta_{2, j, m}+e_{0} w_{j, m} \eta_{1, j, m}\right. \\
\left.+\eta_{1, j, m} \eta_{2, j, m}\right),
\end{gathered}
$$

where $w_{j, m}, \eta_{1, j, m}$, and $\eta_{2, j, m}$ are the $m$-th sample of $w_{j}(t)$, $\eta_{1, j}(t)$ and $\eta_{2, j}(t)$ in the interval $[0, T]$, and $w_{j}(t) \triangleq(b * h *$ $\left.h_{\mathrm{ZF}}\right)\left(t+j T_{\mathrm{f}}+c_{j} T_{\mathrm{p}}\right)=\sqrt{E_{\mathrm{p}}} a_{j} \sum_{l=1}^{L} \alpha_{l} p\left(t-\tau_{l}\right), \eta_{1, j}(t) \triangleq$ $\widetilde{n}\left(t+j T_{\mathrm{f}}+c_{j} T_{\mathrm{p}}-N_{\mathrm{s}} T_{\mathrm{f}}\right)$ and $\eta_{2, j}(t) \triangleq \widetilde{n}\left(t+j T_{\mathrm{f}}+c_{j} T_{\mathrm{p}}\right)$.

As for the case of TR signaling, no assumption on $c_{j}$ is needed for our analysis. For $a_{j}$, we exploit symmetry and consider $a_{j}=+1$ for all $j$. Conditioned on $d_{0}=+1^{4}$, in this case, we can express (21) in the form of (13), where $\beta_{1, j, m}=$ $\frac{1}{2 \sqrt{W}}\left(e_{-1} \eta_{2, j, m}+e_{0} \eta_{1, j, m}\right), \beta_{2, j, m}=\frac{1}{2 \sqrt{W}}\left(e_{-1} \eta_{2, j, m}-\right.$ $\left.e_{0} \eta_{1, j, m}\right)$, and these are statistically independent Gaussian r.v.'s. with variance $\sigma_{\mathrm{DTR}}^{2}=\frac{N_{0}}{4}$. Due to symmetry, we only need to consider $Y_{1}$ and $Y_{2}$ defined in (14), where the noncentrality parameter of $Y_{1}$ is now given by

$$
\mu_{\mathrm{DTR}} \triangleq \frac{1}{2 \sigma_{\mathrm{DTR}}^{2}} \sum_{j=0}^{N_{\mathrm{s}}-1} \sum_{m=1}^{2 W T} \frac{w_{j, m}^{2}}{W}=\frac{2 E_{\mathrm{s}}}{N_{0}} \sum_{l=1}^{L_{\mathrm{CAP}}} \alpha_{l}^{2},
$$

and the pdfs of $Y_{1}$ and $Y_{2}$ conditioned on $\gamma_{\mathrm{DTR}}$ are given by

$$
\begin{aligned}
& f_{Y_{1} \mid \gamma_{\mathrm{DTR}}}\left(y_{1}\right)=f_{\mathrm{NC}}\left(y_{1}, \mu_{\mathrm{DTR}}, q_{\mathrm{DTR}}\right), \\
& f_{Y_{2} \mid \gamma_{\mathrm{DTR}}}\left(y_{2}\right)=f_{\mathrm{C}}\left(y_{2}, q_{\mathrm{DTR}}\right),
\end{aligned}
$$

where $q_{\mathrm{DTR}}=N_{\mathrm{s}} W T$ and $\gamma_{\mathrm{DTR}}=\mu_{\mathrm{DTR}} / 2$. Following (18), the BEP of DTR signaling with AcR is given by

$$
P_{\mathrm{e}, \mathrm{DTR}}=P_{\mathrm{e}}\left(\psi_{\gamma_{\mathrm{DTR}}}(j v), q_{\mathrm{DTR}}\right) .
$$

Comparing (18) and (25), we can observe that the basic difference between TR and DTR signaling lies not only in a doubled non-centrality parameter, but also in a doubled degree of freedom.

For DTR signaling with modified AcR, the new noncentrality parameter of $Y_{1}$ in (14) due to noise averaging in (9) is given by

$$
\begin{aligned}
\mu_{\mathrm{ADTR}} & \triangleq \frac{1}{2 \sigma_{\mathrm{ADTR}}^{2}} \sum_{j=0}^{N_{\mathrm{s}}-1} \sum_{m=1}^{2 W T} \frac{w_{j, m}^{2}}{W} \\
& =\frac{4 N_{\mathrm{s}}}{\left(N_{\mathrm{s}}+1\right)}\left(\frac{N_{\mathrm{s}} E_{\mathrm{p}}}{N_{0}} \sum_{l=1}^{L_{\mathrm{CAP}}} \alpha_{l}^{2}\right),
\end{aligned}
$$

\footnotetext{
${ }^{4}$ When $d_{0}=+1$, the pairs of differentially encoded bits are either $\left(e_{-1}, e_{0}\right)=(+1,+1)$ or $\left(e_{-1}, e_{0}\right)=(-1,-1)$ with probability $\frac{1}{2}$ each. By symmetry, we only need to consider $\left(e_{-1}, e_{0}\right)=(+1,+1)$.
}

where the variance $\sigma_{\mathrm{DATR}}^{2}$ of $\beta_{1, j, m}$ and $\beta_{2, j, m}$ is

$$
\sigma_{\mathrm{ATR}}^{2}=\frac{N_{0}\left(N_{\mathrm{s}}+1\right)}{8 N_{\mathrm{s}}}
$$

and the reduced variance of $\eta_{1, j, m} / \sqrt{W}$ in (21) is $N_{0} / 2 N_{\mathrm{s}}$. The pdfs of $Y_{1}$ and $Y_{2}$ conditioned on $\gamma_{\mathrm{ADTR}}$ are now given by

$$
\begin{aligned}
& f_{Y_{1} \mid \gamma_{\mathrm{ADTR}}}\left(y_{1}\right)=f_{\mathrm{NC}}\left(y_{1}, \mu_{\mathrm{ADTR}}, q_{\mathrm{DTR}}\right), \\
& f_{Y_{2} \mid \gamma_{\mathrm{ADTR}}}\left(y_{2}\right)=f_{\mathrm{C}}\left(y_{2}, q_{\mathrm{DTR}}\right),
\end{aligned}
$$

where $\gamma_{\mathrm{ADTR}}=\mu_{\mathrm{ADTR}} / 2$. Following (18), the BEP of DTR signaling with modified AcR is given by

$$
P_{\mathrm{e}, \mathrm{ADTR}}=P_{\mathrm{e}}\left(\psi_{\gamma_{\mathrm{ADTR}}}(j v), q_{\mathrm{DTR}}\right) .
$$

Note that the analytical framework based on sampling approach can also be used to derive the BEP of TH-PPM signaling with energy detector [18].

\section{Numerical RESUlts}

In this section, we provide some numerical results of both TR and DTR signaling based on our analysis in section III. We consider $N_{\mathrm{s}}=16$ and $L=32$. For UWB channels, it has been verified through experimental results that the fading distribution of the multipath gains can be modeled by the Nakagami- $m$ distribution [3]. As a result, we consider a dense resolvable multipath Nakagami- $m$ fading channel. For simplicity, we consider uniform power dispersion profile (PDP), which serves as a benchmark. Moreover, for such a PDP, the optimum integration interval $T$ is shown to be equal to $L$ [11], [12]. Under uncorrelated scattering assumption, $\left\{\alpha_{l}^{2}\right\}$ are statistically independent and the CF of $\alpha_{l}^{2}$ is given by [15]

$$
\psi_{l}(j v)=\left(\frac{1}{1-\frac{j v}{m L}}\right)^{m},
$$

where the fading severity index $m$ is assumed to be identical for all faded paths.

The BEP performance of TR and DTR signaling with different receiver structures in uncorrelated scattering channels are compared in Fig. 3. The solid and dashed lines indicate the results for TR and DTR signaling respectively. The difference between TR and DTR signaling is about $2 \mathrm{~dB}$, slightly less than the $3 \mathrm{~dB}$ expected from the doubling of the non-centrality parameter in DTR signaling in (22) compared to that of TR signaling in (15). The loss of $1 \mathrm{~dB}$ is associated to the doubled degrees of freedom in DTR signaling (which can be seen by comparing (18) and (25)), which constitutes to more noise accumulation. By comparing the performance between modified AcR and AcR, we can observe that the modified AcR performs better than the AcR by about $3 \mathrm{~dB}$ for both signaling schemes [11], [12]. 


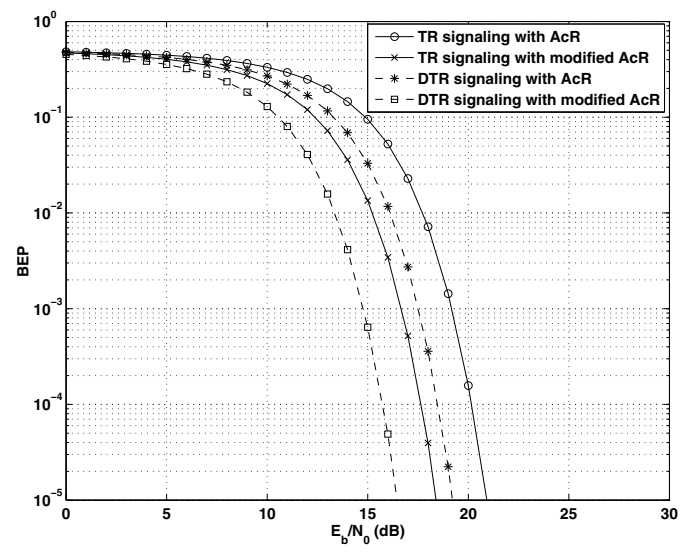

Fig. 3. BEP performance of TR and DTR signaling with AcR and modified AcR in independent Nakagami- $m$ fading channels with uniform PDP, $m=3$. The solid and dashed lines indicate the TR and DTR signaling respectively.

\section{CONCLUSiOnS}

In this paper, we developed an analytical framework and provided a unified performance analysis of TR and DTR signaling for both AcR and modified AcR in dense resolvable multipath channels. Specifically, we derived uncoded BEP of TR and DTR signaling schemes with different receiver structures for a broad class of fading channels, including correlated and uncorrelated scattering channels. The analytical framework is based on the sampling expansion approach, without adopting conventional Gaussian approximation.

\section{ACKNOWLEDGMENT}

This research was supported, in part, by the Charles Stark Draper Laboratory Robust Distributed Sensor Networks Program, the Office of Naval Research Young Investigator Award N00014-03-1-0489, the National Science Foundation under Grant ANI-0335256, and Project VICOM, Ministero dell'Istruzione, Università e della Ricerca Scientific (MIUR), Italy. Davide Dardari would like to thank Prof. Marco Chiani and Prof. Moe Win for giving the opportunity of visiting MIT.

\section{REFERENCES}

[1] M. Z. Win and R. A. Scholtz, "Impulse radio: How it works," IEEE Commun. Lett., vol. 2, no. 2, pp. 36-38, Feb. 1998.

[2] — , "Characterization of ultra-wide bandwidth wireless indoor communications channel: A communication theoretic view," IEEE J. Select. Areas Commun., vol. 20, no. 9, pp. 1613-1627, Dec. 2002.

[3] D. Cassioli, M. Z. Win, and A. F. Molisch, "The ultra-wide bandwidth indoor channel: from statistical model to simulations," IEEE J. Select. Areas Commun., vol. 20, no. 6, pp. 1247-1257, Aug. 2002.

[4] U. G. Schuster and H. Bölcskei, "How different are UWB channels from conventional wideband channels?" Proc. of the International Workshop on Convergent Technol., 2005.

[5] B. Basore, "Noise-like signals and their detection by correlation," Ph.D. dissertation, MIT, Cambridge, MA, May 1952.

[6] C. K. Rushforth, "Transmitted-reference techniques for random or unknown channels," IEEE Trans. Inform. Theory, vol. 10, pp. $39-42$, Jan. 1964.

[7] R. Gagliardi, "A geometrical study of transmitted reference communication system," IEEE Trans. Commun., pp. 118 - 123, Dec. 1964.
[8] A. Polydoros and K. T. Woo, "LPI detection of frequency-hopping signals using autocorrelation techniques," IEEE J. Select. Areas Commun., vol. 3, no. 5, pp. 714 - 726, Sept. 1985.

[9] J. D. Choi and W. E. Stark, "Performance of ultra-wideband communications with suboptimal receivers in multipath channels," IEEE J. Select. Areas Commun., vol. 20, no. 9, pp. 1754 -1766, Dec. 2002.

[10] Y. Chao and R. Scholtz, "Optimal and suboptimal receivers for ultrawideband transmitted reference systems," Proc. IEEE Global Telecomm. Conf., pp. 759-763, Dec. 2003.

[11] T. Q. S. Quek and M. Z. Win, "Ultrawide bandwidth transmittedreference signaling," Proc. IEEE Int. Conf. on Commun., pp. 3409 3413, June 2004.

[12] _ "Analysis of UWB transmitted-reference communication systems in dense multipath channels," IEEE J. Select. Areas Commun., 2005, to be published.

[13] M. Ho, V. S. Somayazulu, J. Foerster, and S. Roy, "A differential detector for an ultra-wideband communications system," Proc. IEEE Semiannual Veh. Technol. Conf., pp. 1896-1900, May 2002.

[14] P. M. Woodward, Probability and Information Theory with Application to Radar. McGraw-Hill, 1953.

[15] M. K. Simon and M.-S. Alouini, Digital Communication over Fading Channels: A Unified Approach to Performance Analysis, 1st ed. New York, NY, 10158: John Wiley \& Sons, Inc., 2000.

[16] F. Ling, "Matched filter-bound for time-discrete multipath Rayleigh fading channel," IEEE Trans. Commun., vol. 43, no. 2/3/4, pp. 710713, Feb./Mar./Apr. 1995.

[17] V. A. Aalo, "Performance of maximal-ratio diversity systems in a correlated Nakagami-fading environment," IEEE Trans. Commun., vol. 43, no. 8, pp. 2360-2369, Aug. 1995.

[18] M. Weisenhorn and W. Hirt, "Robust noncoherent receiver exploiting UWB channel properties," Proc. of IEEE Conference on Ultra Wideband Systems and Technologies (UWBST), pp. 156-160, May 2004. 